Background: Giant cell arteritis (GCA) may be divided into cranial, and extracranial GCA. Tocilizumab (TCZ) has shown efficacy and safety in GCA and other large-vessel vasculitis (LVV) (1-5).

Objectives: To compare the efficacy of TCZ in cranial and extracranial GCA. Methods: Multicenter observational study of 312 patients with GCA treated with TCZ. They were divided into 3 groups a) only cranial (cGCA), b) only extracranial (ecGCA), c) mixed affection (mixGCA). GCA was diagnosed by a) ACR criteria, and/or b) positive temporal artery biopsy, and/or c) LVV by imaging. Remission and sustained remission was defined according to EULAR definitions (1). In ecGCA and mixGCA we also studied the improvement (complete or partial) by imaging techniques.

Results: We studied 312 patients (218 females; mean age, $73.4 \pm 9.6$ years). TABLE shows the main features of the 3 groups. Remission at month 6 was higher in CGCA, as well as the sustained remission at month12 (FIGURE). At 18 and 24 months, were similar in the 3 groups. Improvement by imaging techniques was partial/complete at 6,12,18 and 24 months, in 50\%/0\%,71\%/0\%, 61\%/15\% and $67 \% / 17 \%$ respectively, in ecGCA, and in $75 \% / 0 \%, 53 \% / 18 \%, 64 \% / 12 \%$ and $50 \% / 28 \%$ in mixGCA.

Table 1. Main features of 312 patients at TCZ onset.

\begin{tabular}{|c|c|c|c|c|}
\hline & $\begin{array}{l}\text { Cranial } \\
\text { GCA } \\
(n=152)\end{array}$ & $\begin{array}{c}\text { Extracranial } \\
\text { GCA } \\
(n=49)\end{array}$ & $\begin{array}{l}\text { Mixed } \\
\text { GCA } \\
(n=111)\end{array}$ & $\begin{array}{c}\text { Cranial vs } \\
\text { Extracranial GCA } \\
\text { p }\end{array}$ \\
\hline $\begin{array}{l}\text { Age at TCZ onset, years, mean } \pm \\
\text { SD }\end{array}$ & $76.0 \pm 8.2$ & $65.4 \pm 12.2$ & $73.5 \pm 8.1$ & $0.000^{*}$ \\
\hline Sex, female/male, n (\% female) & $105 / 47(69)$ & $33 / 16(67)$ & $80 / 31(72)$ & 0.960 \\
\hline $\begin{array}{l}\text { Time from diagnosis to TCZ onset } \\
\text { (months, median [IQR] }\end{array}$ & $6[2-21]$ & $7[2-20]$ & 9 [3-25] & 0.765 \\
\hline Biopsy-proven GCA, n (\%) & $87 / 128(68)$ & $0(0)$ & $50 / 87(57)$ & $0.000^{*}$ \\
\hline $\begin{array}{l}\text { Systemic manifestations at } \\
\text { TCZ onset }\end{array}$ & $109(72)$ & $32(65)$ & $84(76)$ & 0.501 \\
\hline Fever, $\mathrm{n}(\%)$ & $18(12)$ & $1(2)$ & $8(7)$ & $0.048^{*}$ \\
\hline Constitutional syndrome, $\mathrm{n}(\%)$ & $52(34)$ & $16(33)$ & $47(42)$ & 0.933 \\
\hline PmR, n (\%) & $88(58)$ & 29 (59) & $71(64)$ & 0.999 \\
\hline $\begin{array}{l}\text { Ischemic manifestations at } \\
\text { TCZ onset }\end{array}$ & $117(77)$ & $0(0)$ & $70(63)$ & $0.000^{*}$ \\
\hline Visual involvement, $\mathrm{n}(\%)$ & $31(20)$ & $0(0)$ & $16(14)$ & $0.000^{*}$ \\
\hline Headache, n (\%) & $103(85)$ & $0(0)$ & $63(57)$ & $0.000^{*}$ \\
\hline Jaw claudication, n (\%) & $39(26)$ & $0(0)$ & $21(19)$ & $0.000^{*}$ \\
\hline \multicolumn{5}{|l|}{ Acute phase reactants } \\
\hline $\mathrm{ESR}, \mathrm{mm} / 1^{\text {st }}$ hour, median [IQR] & 28 [9-53] & 24 [10-43] & $28[15-48]$ & 0.462 \\
\hline $\mathrm{CRP}, \mathrm{mg} / \mathrm{dL}$, median [IQR] & $1.2[0.3-3.4]$ & $0.7[0.4-1.8]$ & $\begin{array}{c}1.6 \\
{[0.4-3.8]}\end{array}$ & 0.153 \\
\hline $\begin{array}{l}\text { Prednisone dose at TCZ onset, } \\
\text { mean } \pm S D\end{array}$ & $26.2 \pm 17.6$ & $15.4 \pm 14.2$ & $20.1 \pm 14.9$ & $0.000^{*}$ \\
\hline $\begin{array}{l}\text { TCZmono/TCZcombo, } \\
\text { n (\% TCZ mono) }\end{array}$ & $116 / 36(76)$ & $26 / 23(53)$ & $69 / 42(62)$ & $0.003^{\star}$ \\
\hline Follow-up (months), mean \pm SD & $27.3 \pm 21.1$ & $32.7 \pm 23.3$ & $27.9 \pm 22.0$ & 0.143 \\
\hline
\end{tabular}

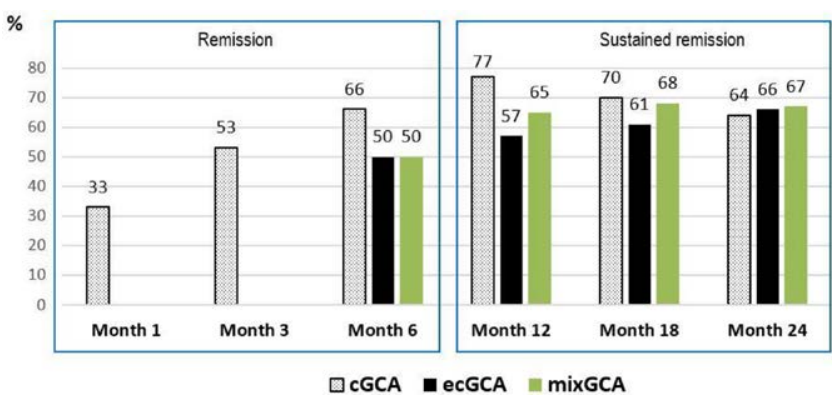

Figure 1. Remission and sustained remission of cGCA, ecGCA and mixGCA according to EULAR (1). In the first 3 months we only could assess cGCA because in ecGCA and mixGCA a control imaging was not performed

Conclusion: TCZ seems to be effective in all phenotypes but it is faster in cGCA in reaching remission. However, improvement by imaging techniques was partial and very rarely complete in ecGCA and mixGCA.

REFERENCES:

[1] Hellmich B, et al. Ann Rheum Dis. 2020; 79: 19-30.

[2] Stone JH, et al. N Engl J Med. 2017; 377: 317-28.

[3] Calderón-Goercke M, et al. Semin Arthritis Rheum 2019; 49:126-35. https:// doi.org/10.1016/j.semarthrit.2019.01.003.

[4] Prieto Peña D et al. Clin Exp Rheumatol 2020 Nov 27. PMID: 33253103.

[5] Loricera J, et al. Clin Exp Rheumatol 2016; 34:S44-53. PMID: 27050507
Disclosure of Interests: Lara Sanchez-Bilbao: None declared, Javier Loricera: None declared, Vicente Aldasoro: None declared, Juan Pablo Valdivieso-Achá: None declared, Ignacio Villa-Blanco: None declared, Olga Maiz: None declared, Rafael Melero: None declared, Clara Moriano: None declared, Julio Sánchez: None declared, Eugenio de Miguel: None declared, Eva Perez-Pampín: None declared, Juan Ramón De Dios: None declared, Juan Carlos Nieto González: None declared, Eva Galíndez-Agirregoikoa: None declared, Patricia Moya: None declared, Francisca Sivera: None declared, José Luis Andréu Sánchez: None declared, Valvanera Pinillos: None declared, Andrea GarcíaValle: None declared, Paloma Vela-Casasempere: None declared, Noelia Alvarez-Rivas: None declared, Marcelino Revenga: None declared, Sara Manrique Arija: None declared, Carlos Fernández-López: None declared, Enrique Raya: None declared, Cristina Hidalgo: None declared, Ruth López-González: None declared, Cristina Campos Fernández: None declared, Antonio Juan-Mas: None declared, Beatriz Arca: None declared, Iñigo Rua-Figueroa: None declared, María Dolors Boquet: None declared, Antonio García: None declared, Adela Gallego: None declared, Eva Salgado-Pérez: None declared, Miguel A GonzálezGay Speakers bureau: Abbvie, Pfizer, Roche, Sanofi, Lilly, Celgene and MSD, Grant/research support from: Abbvie, MSD, Jansen and Roche, Ricardo Blanco Speakers bureau: Abbvie, Lilly, Pfizer, Roche, Bristol-Myers, Janssen, UCB Pharma and MSD, Grant/research support from: Abbvie, MSD and Roche DOI: 10.1136/annrheumdis-2021-eular.2139

\section{OP0065 TOCILIZUMAB IN VISUAL INVOLVEMENT OF GIANT CELL ARTERITIS. MULTICENTER STUDY OF 312 PATIENTS OF CLINICAL PRACTICE}

L. Sanchez-Bilbao ${ }^{1}$, J. Loricera ${ }^{1}$, V. Aldasoro ${ }^{2}$, R. Melero ${ }^{3}$, S. Castañeda ${ }^{4}$ O. Maiz ${ }^{5}$, C. Moriano ${ }^{6}$, I. Villa-Blanco ${ }^{7}$, E. Labrador-Sánchez ${ }^{8}$, C. Hidalgo ${ }^{9}$, S. Manrique Arija ${ }^{10}$, E. Galíndez-Agirregoikoa ${ }^{11}$, E. Perez-Pampín ${ }^{12}$, A. García-Valle ${ }^{13}$, C. Campos Fernández ${ }^{14}, J$. R. De Dios ${ }^{15}$, C. L. Iñíguez ${ }^{16}, J$. L. Andréu Sánchez ${ }^{17}$, J. Sánchez ${ }^{18}$, M. Calderón-Goercke ${ }^{1}$, M. A. GonzálezGay $^{1}$, R. Blanco ${ }^{1}$ on behalf of Tocilizumab in Giant Cell Arteritis Spanish Collaborative Group: Francisca Sivera (HU de Elda), Eugenio de Migue (HU La Paz), Juan Pablo Valdivieso-Achá (H La Princesa), Marcelino Revenga (HU Ramón y Cajal), Guillén Sada (Complejo Hospitalario de Navarra), Carlos Fernández-López (HU Juan Canalejo), Beatriz Arca (HU San Agustín), Ruth López (Complejo Hospitalario de Zamora), Patricia Moya (H Sant Pau), María Dolors Boquet (H Arnau de Vilanova), Adela Gallego (Complejo Hospitalario Universitario de Badajoz), Paloma Vela (H General Universitario de Alicante), Juan Carlos Nieto (HU Gregorio Marañón), Enrique Raya (H San Cecilio), María Noelia Álvarez-Rivas (HU San Agustín), Íñigo Rúa-Figueroa (H Doctor Negrín), Antonio García (H Virgen de Las Nieves), Carlos Vázquez (H Miguel Servet), Arantxa Conesa (HU de Castellón), Carmen Ordás (H Cabueñes), Alejandro Olivé-Marqués (H Trías i Pujol), Carles Galisteo (H Parc Taulí), Francisco Navarro (H General Universitario de Elche), Javier Toyos (HU Virgen Macarena), José Andrés Román Ivorra (HU y Politécnico La Fe), Francisco Ortiz-Sanjuán (HU y Politécnico La Fe), Pau Lluch (H Mateu Orfila), Carmen Torres (Complejo Asistencial de Ávila), Elena Becerra (HU de Torrevieja), Luisa Marena (H La Mancha Centro), Roser Solans Laqué (H Valle de Hebrón), Javier Narváez (H de Bellvitge), Cristina Luna (HU Nuestra Señora de La Candelaria), Nagore Fernández-Llanio (H Arnáu de Vilanova), Susana Romero-Yuste (Complejo Hospitalario Universitario de Pontevedra), Valvanera Pinillos (H San Pedro). ${ }^{1}$ Hospital Universitario Marqués de Valdecilla-IDIVAL, Rheumatology, Santander, Spain; ${ }^{2}$ Complejo Hospitalario de Navarra, Rheumatology, Pamplona, Spain; ${ }^{3}$ Complexo Hospitalario de Vigo, Rheumatology, Vigo, Spain; ${ }^{4}$ Hospital de La Princesa, Rheumatology, Madrid, Spain; ${ }^{5}$ Hospital de Donostia, Rheumatology, San Sebastián, Spain; ${ }^{6}$ Complejo Asistencial Universitario de Leon, Rheumatology, León, Spain; ${ }^{7}$ Hospital Sierrallana, Rheumatology, Torrelavega, Spain; ${ }^{8}$ Hospital SAN PEDRO, Rheumatology, Logroño, Spain; ${ }^{9}$ Complejo Asistencial Universitario de Salamanca, Rheumatology, Salamanca, Spain; ${ }^{10}$ Hospital Regional de Málaga, Rheumatology, Málaga, Spain; ${ }^{11}$ Hospital de Basurto, Rheumatology, Bilbao, Spain; ${ }^{12}$ Hospital Universitario de Santiago, Rheumatology, Santiago de Compostela, Spain; ${ }^{13}$ Complejo asistencial universitario de Palencia, Rheumatology, Palencia, Spain; ${ }^{14}$ Hospital General Universitario de Valencia, Rheumatology, Valencia, Spain; ${ }^{15}$ Hospital del Alto Deba, Rheumatology, Mondragón, Spain; ${ }^{16}$ Hospital Universitario Lucus Augusti, Rheumatology, Lugo, Spain; ${ }^{17} \mathrm{HU}$ Puerta de Hierro, Rheumatology, Madrid, Spain; ${ }^{18}$ Hospital 12 de Octubre, Rheumatology, Madrid, Spain

Background: Visual involvement is one of the most feared complication of Giant Cell Arteritis (GCA).Tocilizumab (TCZ) has shown efficacy and safety in large-vessel vasculitis (LVV) including GCA (1-4).

Objectives: To assess the efficacy of TCZ to: a) prevent the appearance of new ocular involvement, and b) to improve visual symptoms if present. 
Methods: Observational, multicenter study of 312 patients with GCA treated with TCZ. Patients were diagnosed with GCA accordingly to a) ACR criteria, and/or b) biopsy of temporal artery, and/or c) presence of LVV by imaging.

Patients were divided into two subgroups: a) with, and b) without visual involvement at any time. Visual manifestations were classified as: a) Transient visual loss (TVL) (amaurosis fugax), b) Permanent visual loss (PVL) (longer than 24 hours) (partial or complete; unilateral or bilateral), c) diplopia, and d) blurred vision. Accordingly to visual duration up to TCZ onset, we considered: a) 1-10 days, b) 11-30 days, and c) more than 30 days.

Results: We studied 312 (218 women/94 men; mean age73.4+9.6 years) Visual manifestations at any time (before and/or after TCZ) were observed in $78(25 \%)$. In 47 of them visual manifestations were present at TCZ onset, and in the remaining 31 patients had had a complete recovery. Main clinical features of GCA with and without visual involvement are shown in TABLE. Patients with visual involvement were older, with other ischemic complications, and requiring more corticosteroids dose.

Table 1. Main features of $\mathbf{3 1 2}$ patients at TCZ onset.

\begin{tabular}{|c|c|c|c|c|}
\hline & $\begin{array}{l}\text { Overall } \\
\mathrm{N}=312\end{array}$ & $\begin{array}{l}\text { GCA with visual } \\
\text { involvement } \\
(n=78)\end{array}$ & $\begin{array}{l}\text { GCA without } \\
\text { visual } \\
\text { involvement } \\
(n=234)\end{array}$ & p \\
\hline \multicolumn{5}{|l|}{ General features } \\
\hline Age $($ mean $\pm S D)$ & $73.4 \pm 9.6$ & $76.6 \pm 8.0$ & $72.4 \pm 9.8$ & $0.001^{\star}$ \\
\hline Female/Male(\% of female), $\mathrm{n}$ & 218/94 (70) & $47 / 31(60)$ & $171 / 63(73)$ & $0.046^{\star}$ \\
\hline $\begin{array}{l}\text { Time from GCA diagnosis to TCZ } \\
\text { onset (months), median [IQR] }\end{array}$ & 8 [3-24] & $5[1-14]$ & 10 [3-24] & $0.040^{*}$ \\
\hline $\begin{array}{l}\text { Positive TAB, } \mathbf{n}(\%) \\
\text { Ischemic manifestations }\end{array}$ & $137 / 229(60)$ & $33 / 60(55)$ & $104 / 169(61)$ & 0.444 \\
\hline Visual involvement, $\mathrm{n}(\%)$ & $47(15)$ & $47(60)$ & 0 & $0.000^{*}$ \\
\hline Headache, n (\%) & $166(53)$ & $59(76)$ & $107(46)$ & $0.000^{*}$ \\
\hline Jaw claudication & $60(19)$ & $26(33)$ & $34(14)$ & $0.001^{*}$ \\
\hline \multicolumn{5}{|l|}{ Systemic manifestations } \\
\hline Fever, $\mathrm{n}(\%)$ & $27(9)$ & $8(10)$ & $19(8)$ & 0.743 \\
\hline Constitutional syndrome, $\mathrm{n}(\%)$ & $115(37)$ & $30(38)$ & $85(36)$ & 0.878 \\
\hline PmR, n (\%) & $188(60)$ & $46(59)$ & $142(61)$ & 0.830 \\
\hline \multicolumn{5}{|l|}{ Acute phase reactants } \\
\hline ESR, mm/1st hour, median [IQR] & $27[10-50]$ & $34.5[15.2-58]$ & $26.0[10.0-48.0]$ & 0.193 \\
\hline CRP (mg/dL), median [IQR] & $1.4[0.4-3.3]$ & $1.5[0.3-5.5]$ & $1.3[0.4-2.9]$ & 0.134 \\
\hline $\begin{array}{l}\text { Prednisone dose, mg/day, } \\
\text { mean } \pm S D\end{array}$ & $22.3 \pm 16.6$ & $27.1 \pm 18.6$ & $20.8 \pm 15.6$ & $0.008^{\star}$ \\
\hline TCZmono/TCZcombo, n (\%) & $211 / 101$ & $57 / 21$ & $154 / 80$ & 0.295 \\
\hline Follow-up (months), mean $\pm S D$ & $28.4 \pm 21.8$ & $25.8 \pm 22.4$ & $29.3 \pm 21.6$ & 0.119 \\
\hline
\end{tabular}

After TCZ onset, none patient developed new visual involvement. At TCZ onset 47 patients had the following visual manifestations; PVL $(n=28$; unilateral/bilateral; 22/6), TVL ( $n=15$; unilateral/bilateral; 9/6), diplopia $(n=2)$ and blurred vision $(n=2)$.

None of the patients with TVL presented new episodes after TCZ onset, while 8 out of 28 patients with PVL experienced partial improvement (FIGURE). The 2 patients with diplopia and 1 of 2 patients with blurred vision improved.

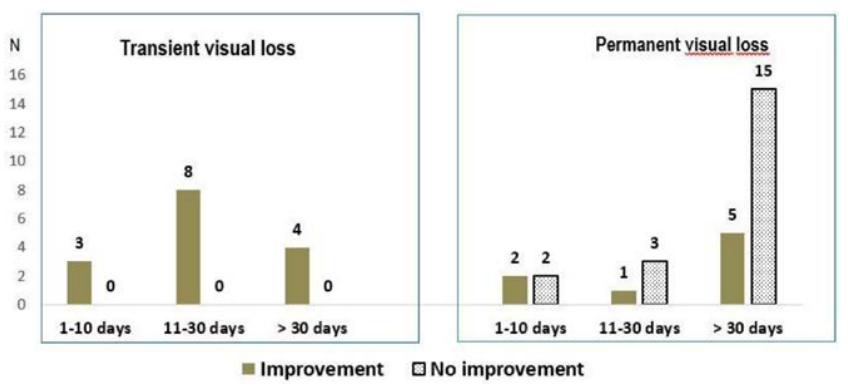

Figure 1. Efficacy of TCZ in 47 patients with GCA and visual involvement at TCZ onset.

Conclusion: TCZ seems to prevent the appearance of new ocular manifestations. When they are present, TCZ may improve totally TVL and partially PVL. REFERENCES:

[1] Stone JH, et al. N Engl J Med. 2017; 377: 317-28.

[2] Calderón-Goercke M, et al. Semin Arthritis Rheum 2019;49:126-35. https:// doi.org/10.1016/j.semarthrit.2019.01.003.

[3] Prieto Peña D et al. Clin Exp Rheumatol 2020 Nov 27. PMID: 33253103.

[4] Loricera J, et al. Clin Exp Rheumatol 2016; 34:S44-53. PMID: 27050507.

Disclosure of Interests: Lara Sanchez-Bilbao: None declared, Javier Loricera: None declared, Vicente Aldasoro: None declared, Rafael Melero: None declared, Santos Castañeda: None declared, Olga Maiz: None declared, Clara Moriano:
None declared, Ignacio Villa-Blanco: None declared, Eztizen Labrador-Sánchez: None declared, Cristina Hidalgo: None declared, Sara Manrique Arija: None declared, Eva Galíndez-Agirregoikoa: None declared, Eva Perez-Pampín: None declared, Andrea García-Valle: None declared, Cristina Campos Fernández: None declared, Juan Ramón De Dios: None declared, Carlota Laura Iñíguez: None declared, José Luis Andréu Sánchez: None declared, Julio Sánchez: None declared, Monica Calderón-Goercke: None declared, Miguel A González-Gay Speakers bureau: Abbvie, Pfizer, Roche, Sanofi, Lilly, Celgene and MSD, Consultant of: Abbvie, Pfizer, Roche, Sanofi, Lilly, Celgene and MSD, Grant/research support from: Abbvie, MSD, Jansen and Roche, Ricardo Blanco Speakers bureau: Abbvie, Lilly, Pfizer, Roche, Bristol-Myers, Janssen, UCB Pharma and MSD, Consultant of: Abbvie, Lilly, Pfizer, Roche, Bristol-Myers, Janssen, UCB Pharma and MSD, Grant/research support from: Abbvie, MSD and Roche DOI: 10.1136/annrheumdis-2021-eular.2169

\section{OP0066 METABOLIC PROFILE AND COMORBIDITIES IN GIANT CELL ARTERITIS AND POLYMYALGIA RHEUMATICA PATIENTS BEFORE AND AFTER TREATMENT}

I. Esen ${ }^{1,2}$, P. Therkildsen ${ }^{3}$, B. Dalsgaard Nielsen ${ }^{4}$, A. Van 't Ende' ${ }^{1}$ A. Boots ${ }^{1,2}$, P. Heeringa ${ }^{2,5}$, E. M. Hauge ${ }^{4}$, E. Brouwer ${ }^{1,2}$, Y. Van Sleen ${ }^{1,2} .{ }^{1}$ University Medical Center Groningen, Rheumatology and Clinical Immunology, Groningen, Netherlands; ${ }^{2}$ University Medical Center Groningen, Vasculitis Expertise Center Groningen, Groningen, Netherlands; ${ }^{3}$ Aarhus University Hospital, Department of Rheumatology, Aarhus, Denmark; ${ }^{4}$ Aarhus University Hospital, Department of Rheumatology and Department of Clinical Medicine, Aarhus, Denmark; ${ }^{5}$ University Medical Center Groningen, Department of Pathology and Medical Biology, Groningen, Netherlands

Background: Giant cell arteritis (GCA) is a granulomatous vasculitis that affects the large vessels, occurring in people over 50 years of age. GCA frequently overlaps with polymyalgia rheumatica (PMR), which affects the proximal joints in the shoulders and hips. Both GCA and PMR patients require long-term treatment with glucocorticoids (GCs). Investigation of new treatment options is required because many GCA and PMR patients experience GC-related adverse events. These include hypercholesterolemia, hypertension, diabetes mellitus, cataract, and infections.(1) Previous studies suggest that unhealthy metabolic features and a higher Body Mass Index (BMI) might be protective in the development of GCA (2). However, data are limited.

Objectives: We aimed to elaborate more-in depth on the metabolic features, BMI and prevalence of comorbidities of GCA and PMR patients at the time of diagnosis and during treatment, to characterize patients for improved treatment options.

Methods: This study included two independent cohorts: the GPS (Groningen) cohort, and the Aarhus cohort for validation of baseline data (Table 1). Patients in the GPS cohort were prospectively followed with visits at 3 months, 1, 2, and 5 years. Laboratory measurements, metabolic co-morbidities were assessed at every visit. At baseline, we assessed whether comorbidities and BMI predicted the duration of GC treatment.

Table 1. Baseline characteristics. P-values were calculated with

Mann-Whitney $U$ test and Fischer Exact test. Numbers in bold: significance between HC and GCA/PMR patients from GPS cohort. *: significance between GCA and PMR of GPS cohort. ${ }^{a}$ : significance between two cohorts.

\begin{tabular}{|c|c|c|c|c|c|}
\hline & $\begin{array}{c}\text { GCA } \\
\text { GPS } \\
\text { cohort } \\
(n=50)\end{array}$ & $\begin{array}{c}\text { PMR } \\
\text { GPS } \\
\text { cohort } \\
(n=44)\end{array}$ & $\begin{array}{c}\text { HCs } \\
\text { GPS } \\
\text { cohort } \\
(n=65)\end{array}$ & $\begin{array}{c}\text { GCA } \\
\text { Aarhus } \\
\text { cohort } \\
(n=52)\end{array}$ & $\begin{array}{c}\text { PMR } \\
\text { Aarhus } \\
\text { cohort } \\
(n=25)\end{array}$ \\
\hline Age, median & 71 & 73 & 70 & 67 & 68 \\
\hline Female, \% & 35 & 26 & 43 & 32 & 13 \\
\hline $\begin{array}{l}\text { Follow-up duration in months, } \\
\text { median }\end{array}$ & 42 & 40 & NA & NA & NA \\
\hline$B M I$, median & 24.3 & 26.6 & 25.4 & 24 & 26.4 \\
\hline Systolic BP (mmHg), median & 140 & 140 & 145 & 135 & 143 \\
\hline Diastolic BP (mmHg), median & 79.5* & 80 & 80 & 76.5 & 83 \\
\hline HbA1c (mmol/mol), median & 43 & 40 & 39 & 43 & 40 \\
\hline CRP (mg/L), median & 54 & 42 & 4 & 76 & 53 \\
\hline ESR $(\mathrm{mm} / \mathrm{h})$, median & $94^{*}$ & 57 & 9 & 77 & 51 \\
\hline Diabetes mellitus, $\%$ & 12 & 23 & 12 & 15 & 8 \\
\hline Hypercholesterolemia, \% & 22 & 14 & 17 & 15 & 24 \\
\hline Hypertension, \% & $66^{a}$ & 55 & 60 & 38 & 36 \\
\hline Cataract, \% & 14 & 16 & 34 & NA & NA \\
\hline
\end{tabular}

Results: Frequencies of metabolic features in GCA/PMR patients from the GPS cohort were not significantly different in comparison to healthy controls (HCs). Higher $\mathrm{HbA} 1 \mathrm{c}$ levels were detected in GCA patients than in HCs. Data on metabolic features from the Aarhus cohort compared well with the GPS cohort data and thus validated these findings (Table 1). Next, the effect of co-existing co-morbidities on the clinical manifestations was investigated. GCA patients with cataract had reduced CRP and ESR whereas PMR patients with cataract had a higher ESR than 